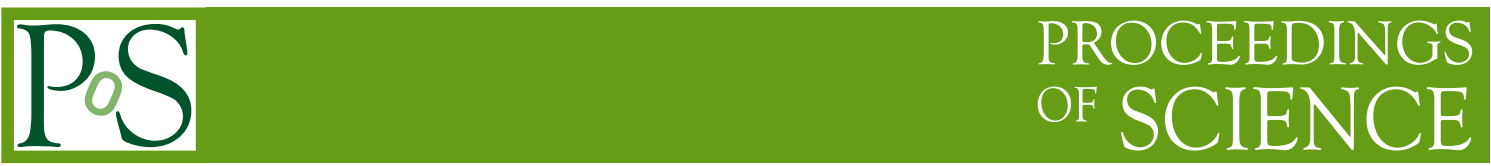

\title{
The search for rare decays of the Higgs boson with ATLAS and CMS
}

\author{
Johannes Elmsheuser*, on behalf of the ATLAS and CMS Collaborations \\ Fakultät für Physik, Ludwig-Maximilians-Universität München, Germany \\ E-mail: johannes.elmsheuser@physik.uni-muenchen.de
}

The Higgs-like boson discovered by the ATLAS and CMS collaborations is a candidate for the last unobserved particle predicted by the Standard Model (SM). The next experimental step is the measurement of its properties, most notably its couplings. This paper presents the searches by ATLAS and CMS for the SM Higgs boson via decays in rare modes such as di-muon pairs, decays to $Z \gamma$ or invisible decays.

The European Physical Society Conference on High Energy Physics -EPS-HEP2013

18-24 July 2013

Stockholm, Sweden

\footnotetext{
* Speaker.
} 


\section{Introduction}

The Standard Model (SM) of particle physics describes exceptionally well nearly all of experimental particle physics data. In the SM, the Brout-Englert-Higgs (BEH) mechanism $[1,2,3]$ spontaneously breaks electroweak (EW) gauge symmetry and generates mass terms for the $W$ and $Z$ gauge bosons and also for the fermion fields via Yukawa couplings. As a consequence of the BEH mechanism, the SM predicts the existence of a heavy scalar particle (the Higgs boson, $H$ ); the search for this particle is a highlight of the Large Hadron Collider (LHC) physics program.

In the summer of 2012 the ATLAS and CMS collaborations discovered a new particle with a mass of approximately $125 \mathrm{GeV}[4,5]$ via decays to photon, $W$ and $Z$ boson pairs with rates consistent with those of the SM Higgs boson. In order to determine whether the newly discovered particle is the SM Higgs boson or a different object, it is fundamental to fully characterize its properties, including parity, spin, and decays to as many final states as possible. This paper presents the search for rare SM Higgs boson decays in three different channels: first in the channel $H \rightarrow Z \gamma$ with $Z \rightarrow \ell^{+} \ell^{-}$where $\ell=e$ or $\mu$ both from ATLAS and CMS, second in the channel of an invisibly decaying SM-like Higgs boson produced in association with a $Z$ boson both from ATLAS and CMS and last in the channel of $H \rightarrow \mu^{+} \mu^{-}$decays from ATLAS. The first two analysis use the 2011 dataset of $7 \mathrm{TeV}$ LHC $p p$ collision data and all analysis use a subset or the full 2012 dataset of $8 \mathrm{TeV}$ LHC $p p$ collision data. All details of the individual analysis discussed below are given in $[6,7,8,9,10,11,12]$ together with prospects of the HL-LHC. The ATLAS and CMS detectors are described elsewhere [13, 14].

\section{Search for $H \rightarrow Z \gamma$ decays}

In the SM, the Higgs boson decays to $Z \gamma$ via loop diagrams similar to $H \rightarrow \gamma \gamma$ decays with the dominant contribution originating from the $W$-mediated amplitudes. The events are selected in $\ell \ell \gamma$ final states with where $\ell=e$ or $\mu$. The initial branching ratio $B R(H \rightarrow Z \gamma)$ is comparable to $B R(H \rightarrow \gamma \gamma)$, but $B R(Z \rightarrow \ell \ell)$ reduces the sensitivity by a factor of 15 . The main backgrounds originate from $Z+\gamma$ events, either from di-boson production in the $\mathrm{t}, \mathrm{u}$ channels (also referred to as initial-state-radiation), from final-state-radiation (FSR) in radiative $Z$ boson decays $(Z \rightarrow \ell \ell \gamma)$ or from parton-to-photon fragmentation, and production of a $Z$ boson in association with jets, followed by a $Z \rightarrow \ell \ell$ decay, and misidentification of a jet as a photon.

The presented analysis use an integrated luminosity of $4.6 \mathrm{fb}^{-1}$ at $\sqrt{s}=7 \mathrm{TeV}$ plus $20.7 \mathrm{fb}^{-1}$ at $\sqrt{s}=8 \mathrm{TeV}$ for ATLAS and $5.0 \mathrm{fb}^{-1}$ at $\sqrt{s}=7 \mathrm{TeV}$ plus $19.6 \mathrm{fb}^{-1}$ at $\sqrt{s}=8 \mathrm{TeV}$ for CMS. The event selection proceeds as following: events with at least two isolated oppositely charged electrons or muons with high transverse momentum $p_{\mathrm{T}}$ and at least one high $p_{\mathrm{T}}$ isolated photon are selected. The angular separation between the photon and one of the leptons has to be $\Delta R(\gamma, \ell)>$ $0.3(0.4)$ ATLAS (CMS). The invariant mass is required to be $m_{\ell \ell \gamma}>m_{Z}-10 \mathrm{GeV}$ (ATLAS) or in the range of $100 \mathrm{GeV}<m_{\ell \ell \gamma}<180 \mathrm{GeV}$ (CMS).

Backgrounds from $Z+\gamma$ (both ISR and FSR) and from $Z+$ jets are estimated from data driven methods, the small contributions from top and $W Z$ backgrounds from Monte Carlo (MC). In the CMS analysis events are further categorized according to the lepton and photon pseudo-rapidity values, in the ATLAS analysis the $\Delta m=m_{\ell \ell \gamma}-m_{Z}$ distribution is used as final distribution since it 
is insensitive to the lepton energy uncertainty and reduces backgrounds from FSR $H \rightarrow \mu \mu$ decays. Fig. 1 shows the $\Delta m$ (ATLAS) and $m_{\ell \ell \gamma}$ (CMS) distributions where the background is fitted with a third order (ATLAS) or fourth and fifth order (CMS) polynomial in data. The main systematic
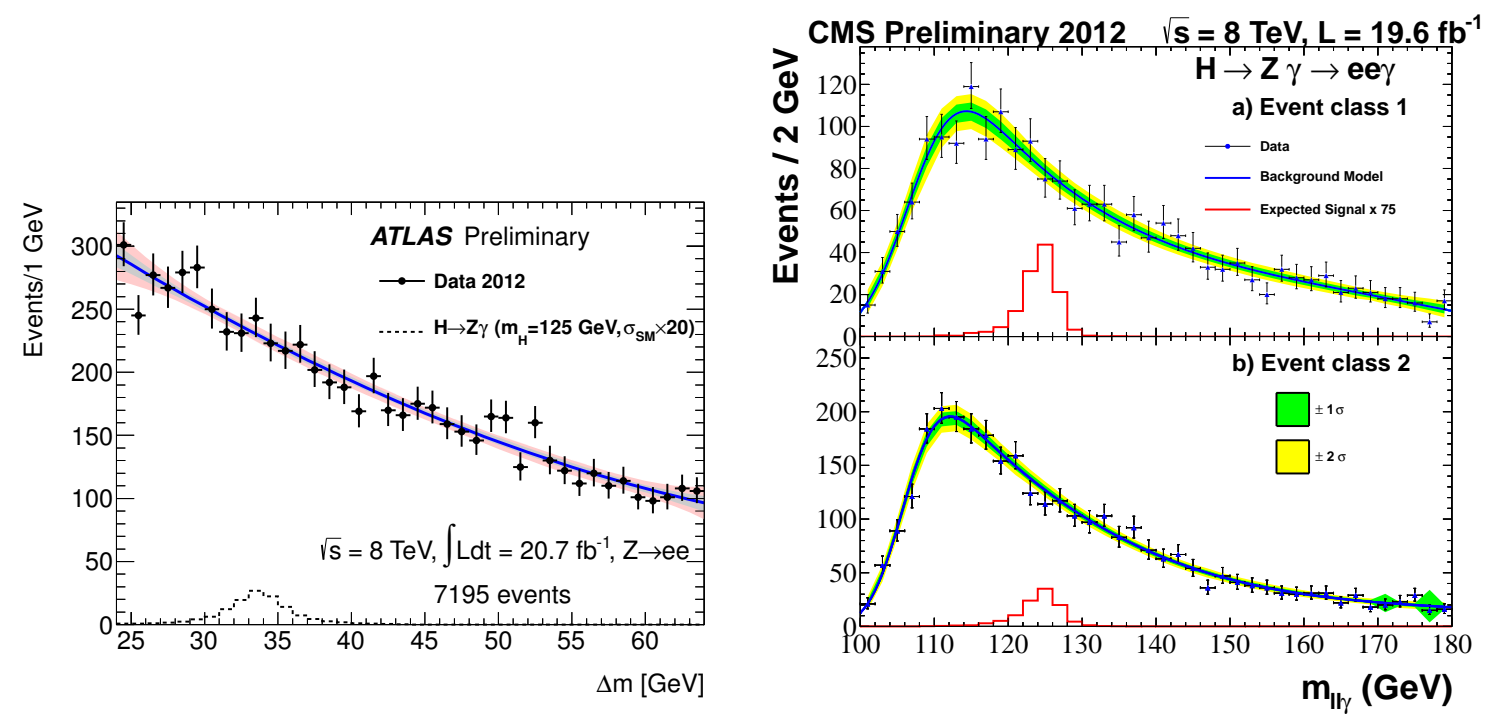

Figure 1: $H \rightarrow Z \gamma$ : Background-only fits to the distribution of the mass difference $\Delta m$ of selected events in ATLAS data [6] (left) and the $m_{\ell \ell \gamma}$ distribution in CMS data [7] (right), both in the $Z \rightarrow e e$ channel at $\sqrt{s}=8$ $\mathrm{TeV}$. Higher order polynomials are used for the fits.

uncertainties are the lepton acceptance of $5 \%$ and the e $/ \gamma$ resolution of 5\%. For CMS the number of predicted signal events in the 2012 dataset for $m_{H}=125 \mathrm{GeV}$ is $6.3(e e)$ and $7.0(\mu \mu)$ events where the number of observed events in the range $120<m_{\ell \ell \gamma}<150 \mathrm{GeV}$ is 5534 and 5993 events, respectively. No excess of events has been observed in the ATLAS and CMS datasets of 2011 and 2012 and limits on the cross section times the branching ration at 95\% CL are derived. The expected limits are in the range of 7.3-22. $\sigma_{S M}$ (ATLAS) and 6-19. $\sigma_{S M}$ (CMS) and the observed limits are in the range of 5.4-37. $\sigma_{S M}$ (ATLAS) and 3-31. $\sigma_{S M}$ (CMS). The expected and observed limits are shown in Fig. 2. Any deviations from the SM prediction will be indication of new electroweak particles coupling to the Higgs boson.

\section{Search for an invisibly decaying SM-like Higgs boson produced in association with a $Z$ boson}

Some extensions to the Standard Model (SM) allow a Higgs boson to decay to stable or longlived particles that interact with the Higgs boson, but have only weak interactions with other elementary particles. Results obtained so far in the search for the SM Higgs boson do not exclude the possibility of a sizable branching ratio to invisible particles for the SM Higgs boson candidate at $m_{H} \approx 125 \mathrm{GeV}$. The signal process searched for is the associated production of $Z H$ where is $Z$ decays leptonically and the Higgs boson is assumed to decay to invisible particles. The analysis searches for an excess of events over the SM contribution in the dilepton + large missing transverse energy $\left(E_{\mathrm{T}}^{\mathrm{miss}}\right)$ final states. Main backgrounds are from $Z Z \rightarrow \ell \ell v v$ decays which contribute $\approx 70 \%$ 

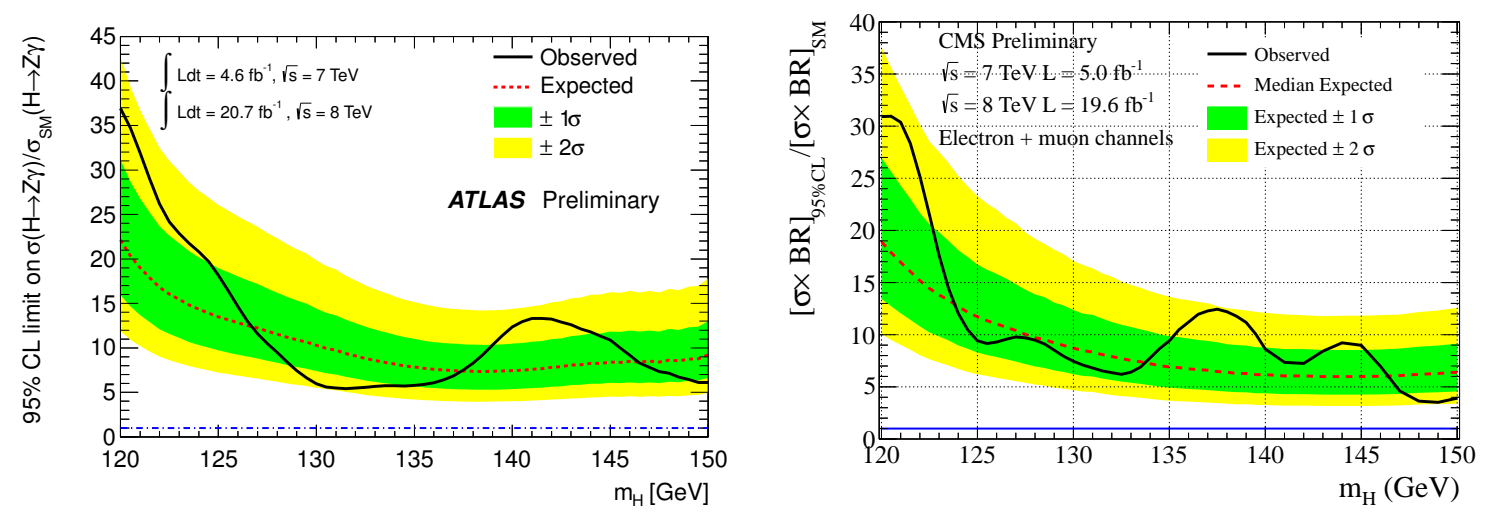

Figure 2: $H \rightarrow Z \gamma$ : Observed 95\%CL limits (solid black line) on the production cross section of a SM Higgs boson decaying to $Z \gamma$, as a function of the Higgs boson mass using $\sqrt{s}=7 \mathrm{TeV}$ and $8 \mathrm{TeV} p p$ collision data from ATLAS [6] (left) and CMS [7] (right). The median expected 95\% CL exclusion limits (dashed red line) are also shown. The green and yellow bands correspond to the $\pm 1 \sigma$ and $\pm 2 \sigma$ intervals.

to the total background, $W Z \rightarrow \ell v \ell \ell$ decays with $\approx 20 \%$ of the total background and $W W \rightarrow \ell v \ell v$ decays with $\approx 5 \%$ of the total background.

The presented analysis use an integrated luminosity of $4.7 \mathrm{fb}^{-1}$ at $\sqrt{s}=7 \mathrm{TeV}$ plus $13.0 \mathrm{fb}^{-1}$ at $\sqrt{s}=8 \mathrm{TeV}$ for ATLAS and $5.1 \mathrm{fb}^{-1}$ at $\sqrt{s}=7 \mathrm{TeV}$ plus $19.6 \mathrm{fb}^{-1}$ at $\sqrt{s}=8 \mathrm{TeV}$ for CMS. The events are selected as follows: events are required to have two isolated high $p_{\mathrm{T}}$ electrons or muons, $\left|m_{Z}-m_{\ell \ell}\right|<15 \mathrm{GeV}, E_{\mathrm{T}}^{\text {miss }}>90 \mathrm{GeV}$ (ATLAS) or reduced $E_{\mathrm{T}}^{\text {miss }}>110 \mathrm{GeV}$ (CMS), an azimuthal opening angle $\Delta \phi\left(E_{\mathrm{T}}^{\text {miss }}, p_{\mathrm{T}}^{\text {miss }}\right)<0.2$ (ATLAS), $\Delta \phi\left(E_{\mathrm{T}}^{\text {miss }}, p_{\mathrm{T}}^{\ell \ell}\right)>2.6, \Delta \phi(\ell \ell)<1.7$ (ATLAS), $\left|E_{\mathrm{T}}^{\text {miss }}-p_{\mathrm{T}}^{\ell \ell}\right| / p_{\mathrm{T}}^{\ell \ell}<0.2$. Events must have no reconstructed jets with $p_{\mathrm{T}}>20 \mathrm{GeV}$ and pseudorapidity $|\eta|<2.5$. Backgrounds from $Z Z$ and $W Z$ decays are determined from MC. $W W$ and top decays are determined from scaling a signal free $e \mu$ decay channel distribution. The backgrounds from $Z+$ jets are estimated from signal and backgrounds enriched and depleted regions (so called $A B C D$ method) using $\Delta \phi\left(E_{\mathrm{T}}^{\text {miss }}, \vec{p}_{\mathrm{T}}^{\text {miss }}\right)$ and the fractional $p_{\mathrm{T}}$ difference (ATLAS) or an reweighted $\gamma+$ jets sample orthogonal to the signal region (CMS). The small backgrounds from $W+$ jets and multi-jet decays are determined from a $4 \times 4$ matrix method with loosened lepton identifications. Fig. 3 shows the distributions of the $E_{\mathrm{T}}^{\text {miss }}$ and reduced $E_{\mathrm{T}}^{\text {miss }}$ for ATLAS and CMS. The dominant systematic uncertainties are from $Z Z$ and $W Z$ background yields with $10-14 \%$ and $Z H$ signal yields with 6-7\%. For CMS the number of predicted signal events in the 2012 dataset for $m_{H}=125$ $\mathrm{GeV}$ is $11.8 \pm 1.9(e e)$ and $16.7 \pm 2.5(\mu \mu)$ events where the number of predicted total background events is $43.1 \pm 4.1(e e)$ and $59.6 \pm 5.5(\mu \mu)$. The number of observed events in 2012 is $33(e e)$ and $45(\mu \mu)$. No excess of events has been observed in ATLAS and CMS data for the 2011 and 2012 datasets and limits on the cross section times the branching ratio at 95\% CL are derived. These limits are interpreted in two ways: branching ratios of $B R(H \rightarrow$ invisible $)>71 \%$ observed $(91 \%$ expected) for CMS and 65\% observed (84\% expected) for ATLAS are excluded at 95\% CL for a SM Higgs boson with $m_{H}=125 \mathrm{GeV}$. Also limits on $\sigma \cdot B R(H \rightarrow$ invisible $)$ for further Higgs-like states in the range of $115<m_{H}<300 \mathrm{GeV}$ are derived. Fig. 4 shows $95 \%$ confidence level limits on the $\mathrm{ZH}$ production cross section multiplied by the invisible branching fraction of such a Higgs boson in the mass range $m_{H}=115 \mathrm{GeV}$ to $m_{H}=300 \mathrm{GeV}$ for the considered data taking periods 

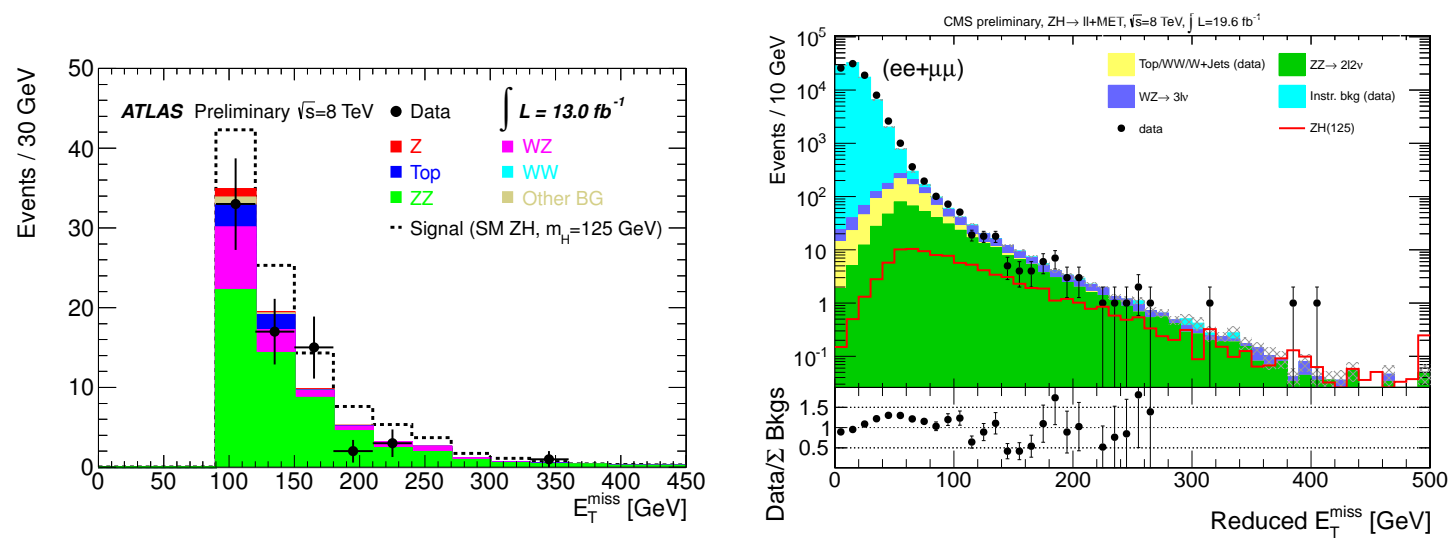

Figure 3: $Z H, H \rightarrow$ invisible: Distributions of $E_{\mathrm{T}}^{\text {miss }}$ for signal events in the 2012 ATLAS data taking period after all cuts [8] (left) and the reduced $E_{\mathrm{T}}^{\text {miss }}$ distribution after the initial lepton selection cuts for CMS [9] (right). The observed data are indicated by the black points and the histograms represent the background predictions. The dashed or red line indicates the prediction from the signal model. The signal model assumes a SM Higgs boson having a mass of $125 \mathrm{GeV}$ and a $100 \%$ branching fraction to invisible particles.

in 2011 and 2012, as well as the limit achieved from the combination of both periods. No excess is observed over the mass range. Stringent limits on Higgs boson and dark matter couplings can
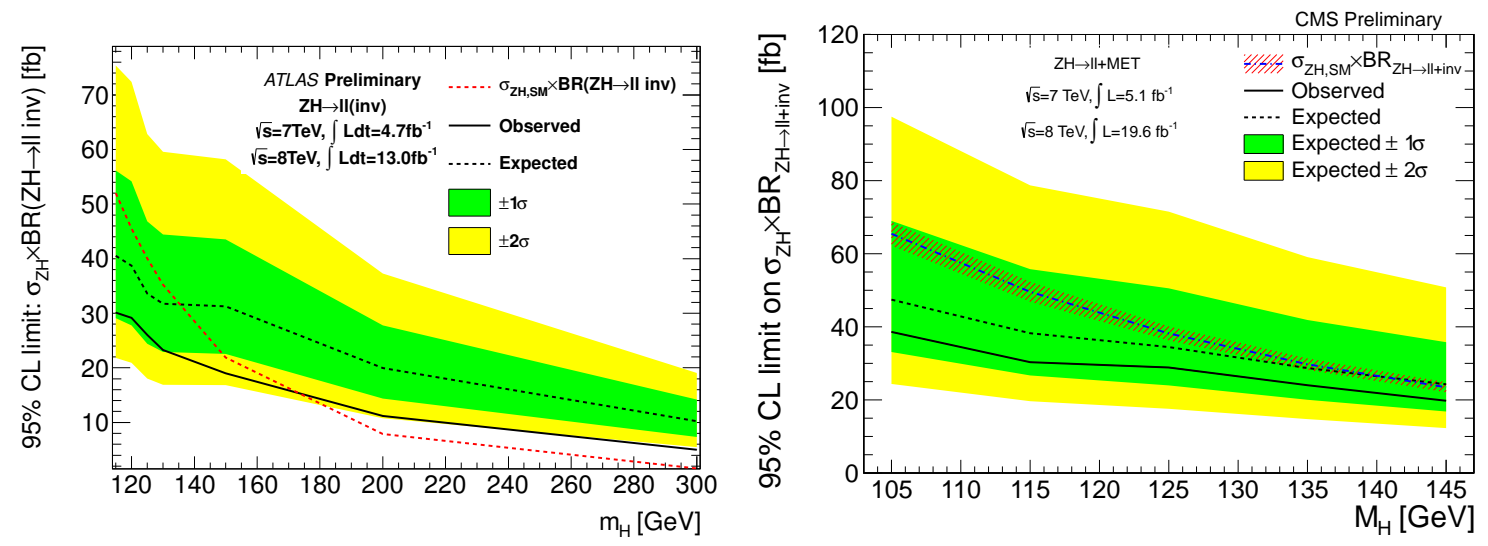

Figure 4: $Z H, H \rightarrow$ invisible: Observed 95\% CL limits (solid black line) on the cross section times branching fraction of a Higgs-like boson decaying to invisible particles as a function of the Higgs boson mass using $\sqrt{s}=7 \mathrm{TeV}$ and $8 \mathrm{TeV} p p$ collision data from ATLAS [8] (left) and CMS [9] (right). The dashed lines show the background only expected limits. The green and yellow bands correspond to the $\pm 1 \sigma$ and $\pm 2 \sigma$ intervals.

be obtained in combination with mono-jet and mono-photon and vector boson fusion produced $H \rightarrow$ invisible searches.

\section{Search for $H \rightarrow \mu^{+} \mu^{-}$decays}

The $H \rightarrow \mu^{+} \mu^{-}$decay has a clean final-state signature and is the only channel where the Higgs coupling to second generation fermions can be measured at the LHC. This is a very challenging measurement due to the small $H \rightarrow \mu^{+} \mu^{-}$branching ratio and high SM backgrounds. The 
dominant irreducible SM background is the $Z / \gamma^{*} \rightarrow \mu^{+} \mu^{-}$process with a very high production rate compared to an expected Higgs signal. The signal is extracted from a fit to the invariant mass $m_{\mu \mu}$ distribution.

The presented analysis uses an integrated luminosity of $20.7 \mathrm{fb}^{-1}$ at $\sqrt{s}=8 \mathrm{TeV}$ for ATLAS. Events are required to have two isolated oppositely charged muons with $p_{\mathrm{T}}^{1(2)}>25(15) \mathrm{GeV}$. The transverse momentum of the di-muon system should have $p_{\mathrm{T}}^{\mu \mu}>15 \mathrm{GeV}$. To increase the sensitivity events are categorized due to the muon momentum resolution with $\left|\eta_{\mu}\right|<1$ and $\left|\eta_{\mu}\right|>1$. The expected number of signal events in a mass window of $10 \mathrm{GeV}$ around $m_{H}=125 \mathrm{GeV}$ are $37.7 \pm 0.2$, where the expected number of total background events is $17700 \pm 130$. The number of observed events in the 2012 dataset if 17442 . Fig. 5 (left) shows the distribution of the invariant mass $m_{\mu \mu}$ after the muon selection cuts.

The dominant systematic uncertainties are from the theoretical signal cross section, signal branching ratio predictions and the experimental muon reconstruction uncertainties. All uncertainties are small and in the order of $1 \%$. The background is fitted in the $m_{\mu \mu}$ distribution with a Breit-Wigner plus exponential function together with a signal function parametrized by a crystal ball + Gaussian function. All fits are validated in MC and data control regions. Since no excess of events is observed, limits on the cross section times branch ratio are derived. The observed and expected 95\% CL limits on the $H \rightarrow \mu^{+} \mu^{-}$signal strength are shown in Fig. 5 (right). The observed (expected) upper limit varies between 3.5 (7.4) and 22.1 (31.3) times the SM prediction in the mass range $110-150 \mathrm{GeV}$. The high luminosity LHC (HL-LHC) will substantially improve the statistical
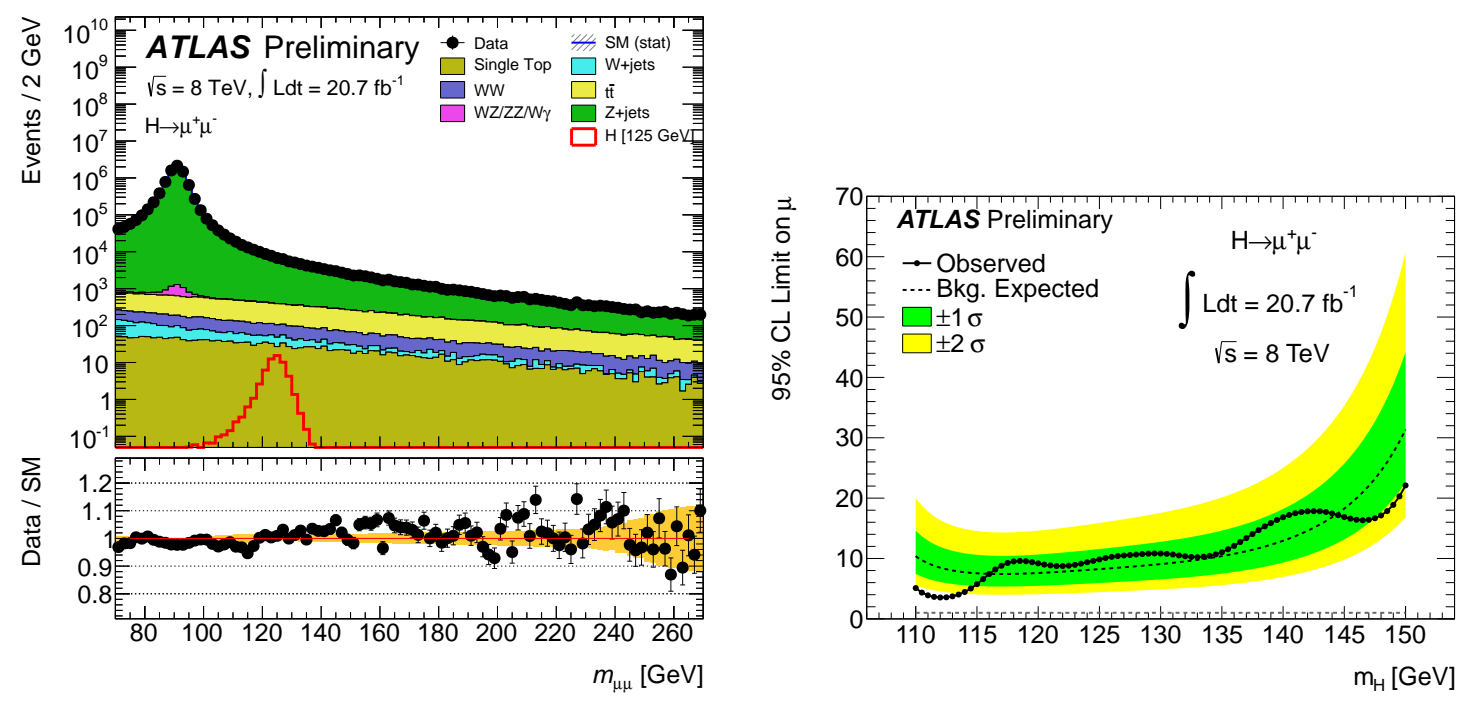

Figure 5: $H \rightarrow \mu^{+} \mu^{-}$, Left: The distribution of the di-muon invariant mass $m_{\mu \mu}$ with the muon selection requirements applied [10]. The signal is shown for $m_{H}=125 \mathrm{GeV}$. The yellow band in the ratio plots shows statistical uncertainty of the MC sample. Right: Observed 95\% CL limits (solid black line) on the cross section times branching fraction of a SM Higgs boson decaying to $\mu^{+} \mu^{-}$as a function of the Higgs boson mass using $8 \mathrm{TeV} p p$ collision data from ATLAS [10]. The dashed line shows the background only expected limits. The green and yellow bands correspond to the $\pm 1 \sigma$ and $\pm 2 \sigma$ intervals.

precision for already established channels and allows to study rare Higgs boson production and decay modes. ATLAS and CMS projects with an integrated luminosity of $3000 \mathrm{fb}^{-1}$ to estab- 
lish a $H \rightarrow \mu^{+} \mu^{-}$signal with an inclusive analysis and an expected significance $>6 \sigma$ (ATLAS) and $\approx 5 \sigma$ for gluon-gluon fusion and vector boson fusion (CMS). The measurement precision is expected to be better than $20 \%$ (ATLAS) or better than $10 \%$ on the $H \mu \mu$ coupling (CMS).

\section{Conclusions}

The LHC, ATLAS and CMS have performed very well during their operation in 2011 and 2012. Searches for a SM Higgs boson in three rare decays channels have been presented. No signal has been observed with the currently analyzed and available data and upper limits on the cross section times branching ratios at a $95 \%$ confidence level have been set. Having not observed any excess over the SM predictions in the discussed channels is a further indication that the observed particle is a SM Higgs boson.

\section{References}

[1] F. Englert and R. Brout, Phys. Rev. Lett. 13 (1964) 321-323.

[2] P. W. Higgs, Phys. Rev. Lett. 13 (1964) 508-509.

[3] G. Guralnik,C. Hagen, and T. Kibble, Phys. Rev. Lett. 13 (1964) 585-587.

[4] ATLAS Collaboration, Phys. Lett. B716 (2012) 1-29, arXiv: 1207.7214 [hep-ex] .

[5] CMS Collaboration, Phys. Lett. B716 (2012) 30-61, arXiv:1207. 7235 [hep-ex] .

[6] ATLAS Collaboration, ATLAS-CONF-2013-009, http://cdsweb.cern.ch/record/1523683.

[7] CMS Collaboration, CMS-PAS-HIG-13-006, https://cds.cern.ch/record/1523674.

[8] ATLAS Collaboration, ATLAS-CONF-2013-011, http://cdsweb.cern.ch/record/1523696.

[9] CMS Collaboration, CMS-PAS-HIG-2013-018, https://cds.cern.ch/record/1561758.

[10] ATLAS Collaboration, ATLAS-CONF-2013-010, http://cdsweb.cern.ch/record/1523695.

[11] ATLAS Collaboration, ATL-PHYS-PUB-2012-004, http://cdsweb.cern.ch/record/1484890.

[12] CMS Collaboration, CMS-NOTE-2012-006, https://cds.cern.ch/record/1494600.

[13] ATLAS Collaboration, JINST 3 (2008) S08003.

[14] CMS Collaboration, JINST 3 (2008) S08004. 